\title{
The social network among the elderly and its relationship with quality of life
}

\author{
Fatemeh Bahramnezhad ${ }^{1}$, Raheleh Chalik ${ }^{2}$, Farideh Bastani ${ }^{3}$, Masoumeh Taherpour ${ }^{4}$, Elham Navab ${ }^{5}$
}

${ }^{1}$ Ph.D. of Nursing, Assistant Professor, Department of Critical Care Nursing, Faculty of Nursing and Midwifery, Tehran University of Medical Sciences, Tehran, Iran

${ }^{2}$ M.Sc., Department of Geriatric Nursing, Faculty of Nursing and Midwifery, Tehran University of Medical Sciences, Tehran, Iran

${ }^{3}$ Ph.D. of Nursing, Associate Professor, Department of Geriatric Nursing, Faculty of Nursing and Midwifery, Iran University of Medical Sciences, Tehran, Iran

${ }^{4}$ M.Sc. in biostatistics, Faculty of Biostatistics, North Khorasan University of Medical Sciences, Bojnoord, Iran

${ }^{5}$ Ph.D. of Nursing, Assistant Professor, Head of Department of Geriatric and Critical Care Nursing, Faculty of Nursing and Midwifery, Tehran University of Medical Sciences, Tehran, Iran

\section{Type of article: Original}

\begin{abstract}
Introduction: Attention to the needs of the elderly is a social necessity, and it seems that evaluating the social network and quality of life of the elderly can be useful in a better understanding of their needs. This study was performed to determine the relationship between the social network and the quality of life of the elderly in the city of Bojnoord in Iran.

Methods: In this cross-sectional study, 201 elderly people aged 60 years or more were selected by continuous and consecutive sampling method in Bojnoord, Iran in 2014. Data were collected using demographic questionnaire, Lubben social network scale and LEIPAD elderly quality of life questionnaire. Data were analyzed by SPSS 16 and using descriptive statistics and independent-samples t-test, ANOVA and Pearson productmoment coefficient.

Results: The results showed that $30.3 \%$ of elderly people studied, were subjected to a high risk of isolation. The highest mean in social network dimensions was in the family $(19.68 \%)$, friends $(12.01 \%)$ and the neighbors $(9.90 \%)$, respectively. The mean score for quality of life of the elderly was as moderate to high $(63.90 \pm 13.73)$, and among the quality of life dimensions, the highest mean was related to the self-care dimension (15.59\%), and the lowest mean was related to the sexual functioning dimension $(1.53 \%)$. The findings suggested a positive and significant relationship between social network and quality of life in the elderly who were studied $(\mathrm{p}<0.000$, $\mathrm{r}=0.468)$.

Conclusion: This study was a step toward understanding the social network status and quality of life of the elderly. It is necessary to say that health care professionals, especially nurses, display a significant role in the community to help people in this regard.
\end{abstract}

Keywords: Aging, Elderly, Social network, Quality of life, Iran

\section{Introduction}

According to the World Health Organization definition, the aging phenomenon indicates the age over 60, and those entering this course of life will experience physiological, psychological and social changes (1). Today, advances in medicine and health have relatively increased the longevity of the human population (2). Regarding these developments as well as social development, the elderly population worldwide and indeed in developing countries, particularly in Iran, is rising. According to the Statistics, annual global population increase is equal to $1.7 \%$, while

\section{Corresponding author:}

Assistant Professor Dr. Elham Navab, Department of Geriatric and Critical Care Nursing, Faculty of Nursing and Midwifery, Tehran University of Medical Sciences, Tehran, Iran.

Tel:+982161054412, E-mail: e-navab@sina.tums.ac.ir, and e_navab100@hotmail.com

Received: July 24, 2016, Accepted: December 24, 2016, Published: May 2017

iThenticate screening: October 01, 2016, English editing: January 08, 2017 , Quality control: February 16, 2017

(C) 2017 The Authors. This is an open access article under the terms of the Creative Commons Attribution-NonCommercialNoDerivs License, which permits use and distribution in any medium, provided the original work is properly cited, the use is non-commercial and no modifications or adaptations are made. 
the elderly population increase is equivalent to $2.5 \%$. According to the latest statistics from the Ministry of Health (2012), 8.2\% of Iran's population comprises of people over 60 years, and it is estimated that this rate of $10.5 \%$ in 2025 will reach to $21.7 \%$ in 2050 (3). Impaired physical functioning is one of the most visible effects of aging, which leads to decreased ability to maintain independence and increased need of help with the onset of aging (4). A research has shown that $58 \%$ of the aged over 65 years are not able to carry out their daily activities, and need help. Due to the debilitating effects of aging, during the treatment and care of the elderly, the factors affecting the quality of their life should also be considered (5). It should also be noted that the caring and therapeutic methods will be useful, only when it can improve the quality of life of the elderly (6). In today's world, the concept of active aging has been introduced; meaning, with the increasing number of the elderly population, their quality of life should also be considered, the aim of supporting the elderly has not be limited only to increase their life expectancy (longevity) (7). Quality of life as one of the most important concepts, has come to the focus of interest of international organizations such as the World Health Organization (WHO) (8). The WHO has defined the quality of life as the individuals' understanding of their situation in life regarding the culture, the value system in which they live, their goals, expectations, standards and priorities (9). Several factors such as health, family, personal relationships, performance in the role, leisure activities and acceptance in social networks affect the quality of life for the elderly (10). Social network is defined as a subjective sense of belonging, being accepted, loved, wanted, respected, and valued and required by someone (11). The individual social network depends on the individual's characteristics and those with whom they interact, and the individual's perception of the support provided for them by network members (12). The family is still considered the best place to ensure comfort and peace for the elderly. The elderly live in the family environment with their spouse, children, friends or their relatives and interact with them. and the important people are very sensitive and effective in the lives and relationships of seniors with others. They could be family members, relatives, friends and acquaintances of the aged; those who are present in the event of problems such as illness, disability, financial or emotional problems as well as at the time of making important decisions in the family, and coming to their aid (13). Impairment in social interactions and isolation, and contradiction in communications are major sources of stress, while supportive social relationships and intimacy cause increased emotional strength (14). Social communications further strengthen self-confidence in the elderly, and strengthening the social networks of the elderly can be a solution to the problem of loneliness in the elderly. Reduced social networks increases the sense of loneliness in the elderly (15), and incoherent social networks are associated with depression, despair and loss of well-being in the elderly, and subsequently affect the quality of life of them (16). To achieve healthy and active aging, all aspects of physical, psychological, social, economic and spiritual health of life need to be considered, and since many diseases and conditions in elderly times are due to unhealthy lifestyles, the foundation of health in these dimensions must be underpinned from the first stages of life, by applying the correct style and improving the quality of life (17). Thus, the increasing number of elderly with rising life expectancy requires more attention to their quality of life. Quality of life concerns most communities, including Iran (18). The above mentioned concerning the importance of social network and quality of life in the elderly indicates the need for research in this context. This study was performed to determine the relationship between social network and the quality of life of the elderly in the city of Bojnoord in Iran.

\section{Material and Methods}

\subsection{Research design and participants}

This study was a cross-sectional study with a sample size of 201 elderly residents in the city of Bojnoord (located in the north east of Iran) in 2014. Sampling was done continuously and sequentially using the convenient sampling method in health care centers, mosques and parks in Bojnoord. Thus, the researcher visited the parks, mosques and community centers seeking elderly people during their leisure time. Sampling was done according to the inclusion criteria.

\subsection{Selection criteria}

Inclusion criteria were those willing to participate, aged 60 and older, the ability to answer the questions and not having cognitive problems or depression, using the Abbreviated Mental Test Score (AMTS). Exclusion criteria were those not wishing to take part in the study.

\subsection{Data collection}

Mental Test Score (AMTS), which consisted of ten items and ten scores (items including age, time of day, year, the name of the hospital or the community, identifying two related persons or employees, date of birth, name of the leader, the ability to count backwards from 20 to 1) and requiring a minimum of seven points, was performed. Then, due to ethical considerations regarding the purposes of the study, the required descriptions were provided for the 
participants, and their participation was attracted to sign a consent form. Then, demographic and profile forms as well as the research tools, including Lubben social network scale and LEIPAD quality of life questionnaires were completed through self-reporting. For illiterate participants, the questionnaires were completed by the researcher in face to face interviews. To determine the quality of life, LEIPAD questionnaire was used. This questionnaire has 31 questions and 7 dimensions. The questionnaire has been initially designed in such a way so that its dimensions would not be specified for the responder. Its dimensions include:

- Physical dimension (five items, questions $1,6,7,9,12$ )

- Self-care dimension (six items, questions $2,3,4,5,10,11$ )

- Depression and anxiety dimension (four items, questions $17,18,19,20$ )

- Mental functioning dimension (five items, questions $8,13,14,15,16$ )

- Social functioning dimension (three items, questions $21,22,23$ )

- Sexual functioning dimension (two items, questions 24, 25)

- Life satisfaction dimension (six items, questions $26,27,28,29,30,31$ )

The questionnaire used 4-point Likert scale items. The minimum score is zero and the maximum is 93 . Its validity and reliability were approved using content validity and Cronbach's alpha as 0.83 . In order to confirm the validity of the instrument, it was given to 10 faculty members in the School of Nursing and Midwifery with a conceptual expertise. After studying and analyzing the faculty members' ideas, the necessary changes were made by researchers.

\subsection{Data analysis}

Data were analyzed by SPSS 16 and using descriptive statistics and independent-samples t-test, ANOVA and Pearson product-moment coefficient.

\section{Results}

The results showed that the age mean and SD of the samples were as $70.02 \pm 6.81$. Also, $43.3 \%$ of participants were male and $56.7 \%$ were female. The living status was as follows: $17.9 \%$ of the elderly with their spouse, $13.4 \%$ with their children, $44.8 \%$ with their spouse and children and $23.9 \%$ of the elderly independently. One of the participants was single $(0.5 \%) ; 35.8 \%$ were married; in $62.2 \%$, the spouse had died, and $1.5 \%$ were divorced. The respondents were asked to describe their unfulfilled needs in life, such as money, housing, and the need for insurance, the need for companionship, whether they need to be accepted in the society, a combination of the items listed, or they do not feel any shortage. In response, $50.2 \%$ of the respondents (101 people) mentioned one or more of the material needs (income, housing and insurance) as the shortage. By examining the social network of elderlies of interest, $32.8 \%$ of elderlies (66 people) were in isolation mode. Also, $30.3 \%$ were at high risk of isolation, $23.9 \%$ were at average risk of isolation and $12.9 \%$ were at low risk of isolation. In sum, more than half of elderlies were in isolation mode and high risk for isolation in terms of social network. Investigating the dimensions of social network showed that the greatest social network mean is related to social network of family with the mean of 19.68. After that, there was social network of friends with the mean of 12.014 and the lowest mean was related to social network of neighbors with the mean of 9.90. All the respondents took the least advantage from social network of family, but for those respondents who did not have any relationship with neighbors and friends; their social network score regarding friends and neighbors was zero. Descriptive investigation of life quality dimensions showed that the greatest life quality mean was related to self-care with the mean of 2.59. Life quality in mental performance with the mean of 2.17 , physical performance of life quality with the mean of 1.99 , and life satisfaction with the mean of 1.84 were the next cases, respectively. The lowest life quality was related to sexual performance with the mean of 0.76 . In sum, since the mean of life quality in the range of 0-3 is 2.06, it is possible to consider life quality of elderlies between average and high. In order to investigate the relationship between life quality and social network, correlation analysis and Pearson product-moment correlation were used. The Pearson product-moment correlation was 0.468. Since the significance level of the test was 0.000 , it can be said that there is a significant direct relationship between life quality and social network of elderlies. The correlation analysis and Pearson product-moment correlation showed that there is a significant positive relationship between all aspects of life quality in the family. Apart from sexual performance, and due to its significance level $(\mathrm{p}=0.370)$, it can be said that there was not any significant relationship between social network in the family and sexual performance. The greatest correlation coefficient of social network in family dimension was with the social performance of life quality with the coefficient of 0.47. There was a significant positive relationship between all dimensions of life quality with the social network, except social performance that, due to the significance level of $(p=0.190)$ it can be said that there is not any significant relationship between social network in neighbors and social performance. The greatest correlation coefficient of social network in neighbors is with sexual performance of life quality as 0.23 . Pearson product-moment correlation 
showed that there is a significant positive relationship between life quality score and all dimensions of social network. The correlation coefficient between life quality and social network was 0.468 which is considered as a significant positive relationship. After that there is a correlation coefficient between social network of family and life quality which is 0.367 . The lowest correlation coefficient between life quality and social network dimensions is related to neighbors, which is 0.2 (Table1).

Table1. The relationship between the dimension of social network and quality of life dimension in elderly

\begin{tabular}{|c|c|c|c|c|c|c|c|}
\hline \multirow{2}{*}{$\begin{array}{l}\text { Social } \\
\text { Network } \\
\text { dimension }\end{array}$} & \multicolumn{7}{|c|}{ Quality of life dimension } \\
\hline & $\begin{array}{l}\text { Physical } \\
\text { functioning }\end{array}$ & $\begin{array}{l}\text { Self- } \\
\text { care }\end{array}$ & $\begin{array}{l}\text { Anxiety \& } \\
\text { Depression }\end{array}$ & $\begin{array}{l}\text { Mental } \\
\text { Function }\end{array}$ & $\begin{array}{l}\text { Social } \\
\text { Performance }\end{array}$ & $\begin{array}{l}\text { Sexual } \\
\text { function }\end{array}$ & $\begin{array}{l}\text { Life } \\
\text { satisfaction }\end{array}$ \\
\hline The family & $\begin{array}{l}r=0.20 \\
p=0.000\end{array}$ & $\begin{array}{l}\mathrm{r}=0.20 \\
\mathrm{p}=0.000\end{array}$ & $\begin{array}{l}\mathrm{r}=0.40 \\
\mathrm{p}=0.000\end{array}$ & $\begin{array}{l}\mathrm{r}=0.32 \\
\mathrm{p}=0.000\end{array}$ & $\begin{array}{l}\mathrm{r}=0.47 \\
\mathrm{p}=0.000\end{array}$ & $\begin{array}{l}r=0.06 \\
p=0.37\end{array}$ & $\begin{array}{l}r=0.39 \\
p=0.000\end{array}$ \\
\hline Neighbors & $\begin{array}{l}r=0.23 \\
p=0.001\end{array}$ & $\begin{array}{l}\mathrm{r}=0.17 \\
\mathrm{p}=0.018\end{array}$ & $\begin{array}{l}\mathrm{r}=0.15 \\
\mathrm{P}=0.04\end{array}$ & $\begin{array}{l}\mathrm{r}=0.15 \\
\mathrm{p}=0.03\end{array}$ & $\begin{array}{l}\mathrm{r}=0.09 \\
\mathrm{P}=0.190\end{array}$ & $\begin{array}{l}r=0.16 \\
p=0.025\end{array}$ & $\begin{array}{l}r=0.20 \\
p=0.002\end{array}$ \\
\hline Friends & $\begin{array}{l}r=0.28 \\
p=0.000\end{array}$ & $\begin{array}{l}r=0.20 \\
p=0.000\end{array}$ & $\begin{array}{l}\mathrm{r}=0.40 \\
\mathrm{p}=0.000\end{array}$ & $\begin{array}{l}\mathrm{r}=0.43 \\
\mathrm{p}=0.000\end{array}$ & $\begin{array}{l}\mathrm{r}=0.30 \\
\mathrm{p}=0.000\end{array}$ & $\begin{array}{l}r=0.23 \\
p=0.000\end{array}$ & $\begin{array}{l}r=0.36 \\
p=0.000\end{array}$ \\
\hline
\end{tabular}

\section{Discussion}

Regarding the determination of elderlies' social network status, the research findings showed that the lowest mean is related to social network of neighbors with the mean of 9.90. In this regard, Netuveli et al. (2005), in a longitudinal study in England on 11,234 elderlies over 65, investigated factors influencing elderlies' life quality. The results of this study showed that having a relationship with the family, friends and living in a good neighborhood with confidence, leads to increased life quality of elderlies (19). The findings of this study point to the importance of having social relationship among elderlies. It seems that changing modern life conditions and people, including elderlies towards industrialization and urban life, leads to the limitation of relationship network and these people are at the risk of isolation. Findings show that the mean of elderlies' social network is lower than average. The results of study by Rutledge et al. (2003) showed that the mean of elderlies' social network in the US is higher than average, which is not consistent with the present study (20). Maybe this inconsistency is due to the differences in designs. It should be noted that in the above study, the Le Bon social network tool (10 questions) was used, but in the present study, the Le Bon social network tool (18 questions) that was used, was more comprehensive, and investigating more dimensions regarding elderlies' social network. In another study by Heidary et al. (2009), the relationship between social support and size of social network with the life quality of patients with cancer was investigated. The social network size was evaluated by asking the patients questions about the people who support them as well as their relationships with others such as sister, mother, friends and others (21). All of these people showed the size of patients' social network. According to the results of the above study, most of these patients considered their family members and spouses as the supporter and member of social network which, from this aspect, is similar to the present study, because the results of the present study showed that elderlies' social network in family dimension has greater mean. Although the tools used in Heidary et al. (2009) are different from the present study and only the size of social network has been evaluated, the importance of family members in the present study, and Heidary et al. (2009), shows the importance of social network, especially family dimension that, from this aspect, the results can be considered as similar and maybe can be related to the importance of role of family in our country and culture. In the case of determining elderlies' life quality in different dimensions including physical, self-care, anxiety and stress, mental performance, social performance, sexual performance, and life satisfaction, the findings of the present study showed that elderlies' life quality has been average to high. In a study by Ahmadi et al. (2004) in Zahedan, elderlies' life quality was investigated using SF-36. The life quality in all aspects (physical and mental) was undesirable, which is inconsistent with the results of the present study. It seems that in the present study, due to using LEIPAD questionnaire, which is considered as a technical questionnaire for elderlies' life quality, the results are more acceptable (22). Meanwhile, the existence of difference between demographic features of samples in these two studies can propose the differences in results, because elderlies' education level in the Ahmadi et al. (2009) study $(80.5 \%$ illiterate) was different from the elderlies in the present study where illiteracy consisted of a lower percentage ( $40.8 \%$ illiterates). Since the education level can influence life quality of elderlies, the education level can be considered as an effective factor in heterogeneity of the results in these two studies. The findings of Lopez et al. (2005) showed that the education level influences life quality of elderly males and females, and elderlies with a higher education level have a higher quality of life. The results of this study are consistent with the present study (23). 
Regarding the determination of the relationship between social network and elderlies' life quality, the findings of the present study showed that there is a significant relationship between life quality and social network of elderlies. The results of Heidari et al. (2008) indicate a significant direct relationship between life quality and social network size of patients with cancer, which is consistent with the present study (21). Although in this study, one open question (naming the supporters by mentioning the relationship with the patient) was used to investigate life quality, the patients were not necessarily elderly and the findings of this study confirm the results of the present study. The findings of Hawton et al. (2011) showed a direct relationship between final feeling and non-uniform social network with depression, hopelessness and decreased welfare among elderlies (18). Although in their study, the relationship between social network and life quality has not been investigated, it seems that the results of this study are consistent with the present study, because the results of Hawton et al. (2011) indicate a direct relationship between social network and depression, hopelessness, and welfare among elderlies that is consistent with the present study, since depression, hopelessness and welfare can influence life quality of elderlies. There was a significant positive relationship between total score of life quality and all dimensions of social network. In a study by Morita (2010), it was observed that decreased relationship with friends is related to decreased life quality (24). Of course, in this study and to evaluate social network, questions about marital status, living with the family or alone, and visiting family and friends were used. But in terms of importance, social network of friends in life quality had the highest consistency between life quality score and social network of friends. The results of a study by De Belvis et al. (2008) indicate a significant direct relationship between visiting friends and increased life quality in physical and mental aspects (25). The results of the above study point to the importance of the role of friends and making connections with them in improving life quality among the elderly.

\section{Conclusions}

The results of the present study show a direct relationship between life quality score and all dimensions of social network that point to the importance of creating efficient social relationship among the elderly and improving their social networks in family, friends and neighbors that bring an improved life quality of elderly people. According to the findings, half of the elderlies were in isolation mode and high risk for isolation in terms of social network. It is suggested to accept elderlies in their respective societies, create specific centers for welfare and local communities to encourage elderlies to voluntarily participate in activities to improve their life quality by improving the quality of social networks.

\section{Acknowledgments:}

This article is part of a research project approved by Tehran University of Medical Sciences. The number was 23403-28-02-92. The authors are grateful to the Vice-president of Research of Nursing and Midwifery Faculty of Tehran University of Medical Sciences and all those who participated in this study.

\section{Conflict of Interest:}

There is no conflict of interest to be declared.

\section{Authors' contributions:}

All authors contributed to this project and article equally. All authors read and approved the final manuscript.

\section{References:}

1) Cromley EK, Wilson-Genderson M, Christman Z, Pruchno RA. Colocation of older adults with successful aging based on objective and subjective measures. Applied Geography. 2015; 56: 13-20. doi: 10.1016/j.apgeog.2014.10.003.

2) Afshar PF, Asgari P, Shiri M, Bahramnezhad F. A Review of the Iran's elderly status according to the census records. Galen Medical Journal. 2016; 5(1): 1-6.

3) Taheri P. Aging population report. Ministry of Health and Medical Education. 2013; 3(3): 26-46.

4) Hasan-poordehbokri A, Masoodi R, Naderipoor A, Pormirza-kalhori R. The effects of exercise on quality of life of elderly people in Shahrekord city. Iran J Elderly. 2007; 2(6): 437-44.

5) Hutchinson A, Rasekaba TM, Graco M, Berlowitz DJ, Hawthorne G, Lim WK. Relationship between health-related quality of life, and acute care re-admissions and survival in older adults with chronic illness. Health Qual Life Outcomes. 2013; 11: 136. doi: 10.1186/1477-7525-11-136. PMID: 23919897, PMCID: PMC3750289. 
6) Giles LC, Hawthorne G, Crotty M. Health-related quality of life among hospitalized older people awaiting residential aged care. Health Qual Life Outcomes. 2009; 7: 71. doi: 10.1186/1477-7525-7-71. PMID: 19630996, PMCID: PMC2725036.

7) Ajh N, Mehrtash B, Javadi A. Effect of education and social support on quality of life among elderly living in nursing homes. J Qazvin Univ Med Sci. 2012; 16(3): 46-52.

8) Asgari P, Bahramnezhad F, Mehrdad N, Noughabi AAA, Hekmatpou D, Mahmoudi M. Depression, functionality and adaptability of elderly patients after open heart surgery off-or on-pump. Jundishapur Journal of Chronic Disease Care. 2015; 4(4): e30508. doi: 10.17795/jjcdc-30508.

9) Pucci G, Reis RS, Rech CR, Hallal PC. Quality of life and physical activity among adults: populationbased study in Brazilian adults. Qual Life Res. 2012; 21(9): 1537-43. doi: 10.1007/s11136-011-0083-5. PMID: 22362520.

10) Huguet N, Kaplan MS, Feeny D. Socioeconomic status and health-related quality of life among elderly people: results from the Joint Canada/United States Survey of Health. Soc Sci Med. 2008; 66(4): 803-10. doi: 10.1016/j.socscimed.2007.11.011. PMID: 18155337.

11) Fu SY, Anderson D, Courtney M, Hu W. The relationship between culture, attitude, social networks and quality of life in midlife Australian and Taiwanese citizens. Maturitas. 2007; 58(3): 285-95. doi: 10.1016/j.maturitas.2007.08.017. PMID: 17942251.

12) Amieva H, Stoykova R, Matharan F, Helmer C, Antonucci TC, Dartigues JF. What aspects of social network are protective for dementia? Not the quantity but the quality of social interactions is protective up to 15 years later. Psychosom Med. 2010; 72(9): 905-11. doi: 10.1097/PSY.0b013e3181f5e121. PMID: 20807876.

13) Eliopoulos C. Gerontological nursing. Lippincott Williams \& Wilkins; 2013.

14) Pinquart M, Sörensen S. Influences of socioeconomic status, social network, and competence on subjective well-being in later life: a meta-analysis. Psychol Aging. 2000; 15(2): 187-224. doi: 10.1037/08827974.15.2.187. PMID: 10879576.

15) Holmén K, Furukawa H. Loneliness, health and social network among elderly people - a follow-up study. Archives of gerontology and geriatrics. 2002; 35(3): 261-74. doi: 10.1016/S0167-4943(02)00049-3.

16) Giordano GN, Björk J, Lindström M. Social capital and self-rated health-a study of temporal (causal) relationships. Soc Sci Med. 2012; 75(2): 340-8. doi: 10.1016/j.socscimed.2012.03.011. PMID: 22537483.

17) Hagedoorn M, Van Yperen NW, Coyne JC, van Jaarsveld CH, Ranchor AV, van Sonderen E, et al. Does marriage protect older people from distress? The role of equity and recency of bereavement. Psychol Aging. 2006; 21(3): 611-20. doi: 10.1037/0882-7974.21.3.611. PMID: 16953722.

18) Hawton A, Green C, Dickens AP, Richards SH, Taylor RS, Edwards R, et al. The impact of social isolation on the health status and health-related quality of life of older people. Qual Life Res. 2011; 20(1): 57-67. doi: 10.1007/s11136-010-9717-2. PMID: 20658322.

19) Netuveli G, Wiggins RD, Hildon Z, Montgomery SM, Blane D. Quality of life at older ages: evidence from the English longitudinal study of aging (wave 1). J Epidemiol Community Health. 2006; 60(4): 357-63. doi: 10.1136/jech.2005.040071. PMID: 16537355, PMCID: PMC2566174.

20) Rutledge T, Matthews K, Lui LY, Stone KL, Cauley JA. Social networks and marital status predict mortality in older women: prospective evidence from the Study of Osteoporotic Fractures (SOF). Psychosom Med. 2003; 65(4): 688-94. doi: 10.1097/01.PSY.0000041470.25130.6C. PMID: 12883123.

21) Heiydari S, Salahshorian A, Rafie F, Hoseini F. Correlation of perceived social support and size of social network with quality of life dimension in cancer patients. Feyz Journals of Kashan University of Medical Sciences. 2008; 12(2): 15-22.

22) Ahmadi F, Salar A, Faghihzadeh S. Quality of life in Zahedan elderly population. Hayat. 2004; 10(3): 61-7.

23) García EL, Banegas JR, Pérez-Regadera AG, Cabrera RH, Rodríguez-Artalejo F. Social network and health-related quality of life in older adults: a population-based study in Spain. Qual Life Res. 2005; 14(2): 511-20. doi: 10.1007/s11136-004-5329-z. PMID: 15892440.

24) Morita A, Takano T, Nakamura K, Kizuki M, Seino K. Contribution of interaction with family, friends and neighbours, and sense of neighbourhood attachment to survival in senior citizens: 5-year follow-up study. Soc Sci Med. 2010; 70(4): 543-9. doi: 10.1016/j.socscimed.2009.10.057. PMID: 19944506.

25) de Belvis AG, Avolio M, Spagnolo A, Damiani G, Sicuro L, Cicchetti A, et al. Factors associated with health-related quality of life: the role of social relationships among the elderly in an Italian region. Public health. 2008; 122(8): 784-93. doi: 10.1016/j.puhe.2007.08.018. PMID: 18374375. 